\title{
Orlicz-Pettis Theorems for Multiplier Convergent Series
}

\author{
C. Swartz and C. Stuart
}

\begin{abstract}
Orlicz-Pettis theorems have been established for multiplier convergent series where the multipliers come from $c_{0}$ or $l^{p}(0<p<\infty)$. We show that these results as well as the classical Orlicz-Pettis theorem are corollaries of a general result based on the fact that all of these multiplier spaces have the canonical unit vectors as a Schauder basis.
\end{abstract}

Keywords: Orlicz-Pettis theorems, multiplier convergent series

AMS subject classification: 46 A 03, 46 A 35, 46 A 45

\section{Introduction}

Let $\lambda$ be a scalar sequence space which contains the subspace of all sequences which are eventually 0 , and let $X$ be a locally convex Hausdorff topological vector space. A series $\sum x_{i}$ is said to be $\lambda$ multiplier convergent in $X$ if the series $\sum t_{i} x_{i}$ converges in $X$ for every $t=\left\{t_{i}\right\} \in \lambda$; the elements of the space $\lambda$ are referred to as multipliers. For example, when $\lambda$ is the space $m_{0}$ of all sequences with finite range, $\lambda$ multiplier convergence is just subseries convergence. The classical Orlicz-Pettis theorem for locally convex spaces asserts that a series which is subseries convergent in the weak topology $\sigma\left(X, X^{\prime}\right)$ of $X$ is actually subseries convergent in the original (or Mackey) topology of $X$ [7]. More generally, we refer to a theorem as an Orlicz-Pettis theorem if the result asserts that a series which is $\lambda$ multiplier convergent with respect to the weak topology $\sigma\left(X, X^{\prime}\right)$ is actually $\lambda$ multiplier convergent in some stronger locally convex topology on $X$.

Several Orlicz-Pettis type theorems have recently been established for multiplier convergent series where the multipliers come from some of the classical sequence spaces $c_{0}$ or $l^{p}(0<p<\infty)$ (see [6: Theorem 6] and [13: Theorem 3]). The proofs in these papers are quite different for the cases $1 \leq p<\infty$ and $0<p<1$. In this note we point out that these multiplier convergent results as well as the classical Orlicz-Pettis theorem and some of its generalizations are corollaries of a general result which is based on the fact that all of these multiplier sequence spaces have the canonical unit vectors as a Schauder basis for an appropriate locally convex topology (i.e. they are $A K$-spaces).

C. Swartz: New Mexico State Univ., Dept. Math. Sci., Las Cruces, NM 88003, USA

C. Stuart: Eastern New Mexico Univ., Dept. Math. Sci., Portales, NM 88130, USA

ISSN 0232-2064 / \$2.50 (C) Heldermann Verlag Berlin 


\section{Main results}

We begin by fixing some notation which will be used throughout the sequel. Let $\lambda$ be a vector space of real-valued sequences which contains all of the sequences which are eventually 0 . The $\beta$-dual $\lambda^{\beta}$ of $\lambda$ is the space of all sequences $s=\left\{s_{i}\right\}$ such that $\sum_{i=1}^{\infty} s_{i} t_{i}$ converges for every $t=\left\{t_{i}\right\} \in \lambda ;$ if $s \in \lambda^{\beta}$ and $t \in \lambda$, we write $s \cdot t=\sum_{i=1}^{\infty} s_{i} t_{i}$ and note that $\lambda$ and $\lambda^{\beta}$ are in duality with respect to the bilinear map $s \cdot t$. If $E$ and $E^{\prime}$ are two vector spaces in duality with respect to a bilinear mapping, we denote the weak and strong topologies on $E$ from this pairing by $\sigma\left(E, E^{\prime}\right)$ and $\beta\left(E, E^{\prime}\right)$, respectively. Other notation and terminology should be standard.

Let $(X, \tau)$ be a Hausdorff locally convex topological vector space and let $\sum x_{i}$ be a series with $x_{i} \in X$. The series $\sum x_{i}$ is said to be $\lambda$ multiplier convergent with respect to $\tau$ (or $\tau \lambda$ multiplier convergent) if the series $\sum_{i=1}^{\infty} t_{i} x_{i}$ is $\tau$-convergent for every $t \in \lambda$.

Now let $E$ and $E^{\prime}$ be in duality with respect to the bilinear map $\langle\cdot, \cdot\rangle$ and let $\sum x_{i}$ be a $\lambda$ multiplier convergent series in $E$ with respect to $\sigma\left(E, E^{\prime}\right)$. If $t \in \lambda$, let

$$
\sum_{i=1}^{\infty} t_{i} x_{i}=\sigma\left(E, E^{\prime}\right)-\lim _{n} \sum_{i=1}^{n} t_{i} x_{i}
$$

and define a linear map $S: \lambda \rightarrow E$ by $S t=\sum_{i=1}^{\infty} t_{i} x_{i}$. We have the following continuity property for $S$.

Theorem 2.1. The map $S$ is $\sigma\left(\lambda, \lambda^{\beta}\right)-\sigma\left(E, E^{\prime}\right)$ continuous.

Proof. Let $\left\{t^{\delta}\right\}$ be a net in $\lambda$ which is $\sigma\left(\lambda, \lambda^{\beta}\right)$ convergent to 0 and $x^{\prime} \in E^{\prime}$. Since $\sum_{i=1}^{\infty} t_{i}\left\langle x^{\prime}, x_{i}\right\rangle$ converges for every $t \in \lambda,\left\{\left\langle x^{\prime}, x_{i}\right\rangle\right\} \in \lambda^{\beta}$ and

$$
\sum_{i=1}^{\infty} t_{i}^{\delta}\left\langle x^{\prime}, x_{i}\right\rangle=\left\langle x^{\prime}, S t^{\delta}\right\rangle \rightarrow 0 .
$$

That is, $S t^{\delta} \rightarrow 0$ with respect to $\sigma\left(E, E^{\prime}\right)$

Following Wilansky [11: Definition 11.1.5] we say that any polar topology $w\left(E, E^{\prime}\right)$ on $E$ is a Hellinger-Toeplitz topology if whenever $F$ and $F^{\prime}$ are a dual pair and $u: E \rightarrow F$ is $\sigma\left(E, E^{\prime}\right)-\sigma\left(F, F^{\prime}\right)$ continuous, then $u$ is $w\left(E, E^{\prime}\right)-w\left(F, F^{\prime}\right)$ continuous. For example, the Mackey and the strong topologies are Hellinger-Töplitz topologies.

From Theorem 2.1 we have the following

Corollary 2.2. The map $S: \lambda \rightarrow E$ is continuous for any Hellinger-Töplitz topology on the dual pairs $\left(\lambda, \lambda^{\beta}\right)$ and $\left(E, E^{\prime}\right)$.

If $\tau$ is any locally convex topology on $\lambda$, we say that $\lambda$ is an $A K$-space or has the $A K-$ property with respect to $\tau$ if the coordinate functions $t \rightarrow t_{k}$ are continuous and $t=\tau-\lim _{n} \sum_{i=1}^{n} t_{i} e^{i}$ for every $t \in \lambda$, where $e^{i}$ is the canonical unit vector with 1 in the $i^{\text {th }}$ coordinate and 0 in the other coordinates (i.e., the $\left\{e^{i}\right\}$ are a Schauder basis for $\lambda$ with respect to $\tau$ ).

From Corollary 2.2 we obtain the following 
Corollary 2.3 (Orlicz-Pettis). Suppose $w\left(\lambda, \lambda^{\beta}\right)$ is a Hellinger-Töplitz topology on $\lambda$, and $\lambda$ is an $A K$-space with respect to $w\left(\lambda, \lambda^{\beta}\right)$. If $\sum x_{i}$ is $\lambda$ multiplier convergent with respect to $\sigma\left(E, E^{\prime}\right)$, then $\sum x_{i}$ is $\lambda$ multiplier convergent with respect to $w\left(E, E^{\prime}\right)$.

Proof. Let $t \in \lambda$. Since $\sum_{i=1}^{n} t_{i} e^{i} \rightarrow t$ in $w\left(\lambda, \lambda^{\beta}\right)$,

$$
S\left(\sum_{i=1}^{n} t_{i} e^{i}\right)=\sum_{i=1}^{n} t_{i} x_{i} \rightarrow \sum_{i=1}^{\infty} t_{i} x_{i}
$$

in $w\left(E, E^{\prime}\right)$ by Corollary 2.2

The following special case of Corollary 2.3 covers many examples.

Corollary 2.4 (Orlicz-Pettis). Suppose that $\lambda$ has a locally convex topology under which $\lambda$ is barrelled and an $A K$-space. If $\sum x_{i}$ is $\lambda$ multiplier convergent with respect to $\sigma\left(E, E^{\prime}\right)$, then $\sum x_{i}$ is $\lambda$ multiplier convergent with respect to $\beta\left(E, E^{\prime}\right)$.

Proof. $\lambda^{\prime}=\lambda^{\beta}$ by [4: Proposition 3.9] so the original topology of $\lambda$ is $\beta\left(\lambda, \lambda^{\beta}\right)$ and the result follows from Corollary 2.3

We give a number of examples covered by Corollary 2.4 .

Example 2.5. If $\lambda$ is a Banach [Frechet] $A K$-space, Corollary 2.4 applies; e.g. $\lambda=c_{0}, l^{p}(1 \leq p<\infty), c s, b v_{0}[\lambda=\omega[11$ : Section 1.2], a Köthe echelon space [5: Section 30.8]]. In particular, $\lambda=c_{0}$ or $\lambda=l^{p} \quad(1 \leq p<\infty)$ gives the multiplier convergent result of [6: Theorem 6]. There are many examples of Frechet $A K$-spaces. If $A$ is an infinite matrix, denote by $\omega_{A}$ the linear space of all sequences that $A$ maps into $\omega$, the space of all sequences. It is shown in [12: Theorems 4.3 .8 and 4.3.12] that $\omega_{A}$ is a Frechet $A K$-space. Further, $\left(l^{p},\|\cdot\|_{1}\right)(0<p<1)$ is a barrelled $A K$-space [1] so if $\lambda=l^{p} \quad(0<p<1)$, Corollary 2.4 gives the multiplier convergent result of [13: Theorem 3]. Similarly, Corollary 2.4 applies to any barrelled subspace of $\left(l^{1},\|\cdot\|_{1}\right)$; examples of such barrelled subspaces are given in [8: Section 4]. Further examples of barrelled $A K$-spaces are given in the corollary to [2: Theorem 4].

We next show that the classical Orlicz-Pettis theorem for subseries convergent series follows from Corollary 2.3. A series $\sum x_{i}$ in a topological vector space $X$ is subseries convergent if the subseries $\sum x_{n_{i}}$ converges in $X$ for every subsequence $\left\{x_{n_{i}}\right\}$ of $\left\{x_{i}\right\}$. If $m_{0}$ is the space of all sequences with finite range, then a series $\sum x_{i}$ is subseries convergent in $X$ if and only if $\sum x_{i}$ is $m_{0}$ multiplier convergent.

The space $m_{0}$ is not an $A K$-space under its natural topology given by the sup-norm, but it is an $A K$-space under a locally convex topology introduced by $\mathrm{P}$. Dierolf to treat Orlicz-Pettis theorems. We describe Dierolf's topology [3]. Let $\mathcal{M}$ be the family of all subsets $M \subset E^{\prime}$ such that $M$ is $\sigma\left(E^{\prime}, E\right)$-bounded and for every linear, continuous map

$$
T:\left(E^{\prime}, \sigma\left(E^{\prime}, E\right)\right) \rightarrow\left(l^{1}, \sigma\left(l^{1}, m_{0}\right)\right)
$$

$T(M)$ is relatively compact in $\left(l^{1},\|\cdot\|_{1}\right)$. The Dierolf topology on $\delta_{1}\left(E, E^{\prime}\right)$ on $E$ is the polar topology on $E$ of uniform convergence on the elements of $\mathcal{M}$ (see [11: Section 8.5] and [5: Section 21.1]). The topology $\delta_{1}\left(E, E^{\prime}\right)$ is stronger than the Mackey topology and Dierolf shows that it is the strongest polar topology on $E$ such that a series in $\sigma\left(E, E^{\prime}\right)$ subseries convergent if and only if it is $\delta_{1}\left(E, E^{\prime}\right)$ subseries convergent ([3: Theorem 2.2]; see also [3: Corollary 2.4] for a comparison with other polar topologies).

The following property is easily checked (see [11: Theorem 11.2.2]). 
Proposition 2.6. $\delta_{1}\left(E, E^{\prime}\right)$ is a Hellinger-Töplitz topology.

We show that $m_{0}$ is an $A K$-space under the topology $\delta_{1}\left(m_{0}, l^{1}\right)$. Actually, we establish an even stronger result.

Proposition 2.7. For $t \in m_{0}$,

$$
\delta_{1}\left(m_{0}, l^{1}\right)-\lim _{n} \sum_{k=1}^{n} t_{k} e^{k}=t
$$

uniformly for $\|t\|_{\infty} \leq 1$. In particular, $\left(m_{0}, \delta_{1}\left(m_{0}, l^{1}\right)\right)$ is an AK-space.

Proof. If $M \in \mathcal{M}$ (relative to the duality between $m_{0}$ and $l^{1}$ ), then $M$ is relatively compact in $\left(l^{1},\|\cdot\|_{1}\right)$ so $\lim _{n} \sum_{k=n}^{\infty}\left|s_{k}\right|=0$ uniformly for $s \in M$ [9: Theorem 10.1.15]. Thus, for $s \in M$ and $t \in m_{0}$ with $\|t\|_{\infty} \leq 1$ we have $\left|\sum_{k=n}^{\infty} s_{k} t_{k}\right| \leq \sum_{k=n}^{\infty}\left|s_{k}\right|$ and the result follows

From Corollary 2.2 and Proposition 2.6, we obtain a sharpened form of the OrliczPettis theorem for subseries convergent series.

Corollary 2.8 (Orlicz-Pettis). If $\sum x_{k}$ is $\sigma\left(X, X^{\prime}\right)$ subseries convergent, then

$$
\delta_{1}\left(X, X^{\prime}\right)-\lim _{n} \sum_{k=1}^{n} t_{k} x_{k}=\sum_{k=1}^{\infty} t_{k} x_{k}
$$

uniformly for $t \in m_{0}$ with $\|t\|_{\infty} \leq 1$. In particular, $\sum x_{k}$ is $\delta_{1}\left(X, X^{\prime}\right)$ subseries convergent.

The last statement in Corollary 2.8 is Dierolf's version of the classical Orlicz-Pettis theorem for subseries convergent series. Dierolf shows that $\delta_{1}$ is the strongest polar topology for the pair $\left(X, X^{\prime}\right)$ such that the conclusion of the Orlicz-Pettis theorem holds.

The uniform $\delta_{1}$ convergence in Corollary 2.8 seems to be a new observation. By restricting the $t$ 's in $m_{0}$ to be sequences of 0 's and 1 's, the uniform convergence conclusion implies, in particular, that $\sum x_{k}$ is $\delta_{1}$ unordered convergent [10: Proposition 10.1.1].

If $\sum x_{i}$ is subseries convergent, it is known that the set of "partial sums" $\left\{\sum_{i \in \sigma} x_{i}\right.$ : $\sigma \subset \mathbb{N}\}$ is compact [10: Theorem 10.1.3]. We can use Corollary 2.8 to show that a set of partial sums using the larger set of multipliers $\left\{t \in m_{0}:\|t\|_{\infty} \leq 1\right\}$ is precompact.

Proposition 2.9. If $\sum x_{k}$ is $\sigma\left(X, X^{\prime}\right)$ subseries convergent, then

$$
\mathcal{S}=\left\{\sum_{k=1}^{\infty} t_{k} x_{k}: t \in m_{0} \text { with }\|t\|_{\infty} \leq 1\right\}
$$

is $\delta_{1}\left(X, X^{\prime}\right)$ precompact.

Proof. Let $U$ be a $\delta_{1}\left(X, X^{\prime}\right)$ neighborhood of 0 and pick a $\delta_{1}\left(X, X^{\prime}\right)$ neighborhood $V$ of 0 such that $V+V \subset U$ : By Corollary 2.8, there exists $N \in \mathbb{N}$ such that $\sum_{k=n}^{\infty} t_{k} x_{k} \in$ $V$ for $n \geq N$ and $t \in m_{0}$ with $\|t\|_{\infty} \leq 1$. The set

$$
F=\left\{\sum_{k=1}^{l} t_{k} e^{k}:\|t\|_{\infty} \leq 1 \text { and } l \leq N\right\}
$$


is precompact in $\delta_{1}\left(m_{0}, l^{1}\right)$ since $F$ is contained in a finite-dimensional subspace of $m_{0}$, is $\|\cdot\|_{\infty}$ bounded, and $\delta_{1}\left(m_{0}, l^{1}\right)$ is weaker than $\|\cdot\|_{\infty}$. Therefore, $S(F)$ is $\delta_{1}\left(X, X^{\prime}\right)$ precompact by Corollary 2.2. Hence, there exist $z_{1}, \ldots, z_{m} \in F$ such that

$$
S(F) \subset \bigcup_{i=1}^{m}\left(S z_{i}+V\right)
$$

Let $t \in m_{0}$ with $\|t\|_{\infty} \leq 1$. Then there exists $j$ such that $\sum_{i=1}^{N} t_{i} x_{i} \in S z_{j}+V$ so

$$
S t=\sum_{k=1}^{\infty} t_{k} x_{k}=\sum_{k=1}^{n} t_{k} x_{k}+\sum_{k=n+1}^{\infty} t_{k} x_{k} \in S z_{j}+V+V \subset S z_{j}+U .
$$

Hence, $\mathcal{S} \subset \bigcup_{i=1}^{m}(S z ;+U)$ and $\mathcal{S}$ is $\delta_{1}\left(X, X^{\prime}\right)$ precompact

A series which is $l^{\infty}$ multiplier convergent is often referred to as a bounded multiplier convergent series. We next show that Corollary 2.3 can be used to establish an OrliczPettis theorem for bounded multiplier convergent series. Again, $l^{\infty}$ is not an AK space under the sup-norm, but it is an $A K$-space under another locally convex topology also introduced by Dierolf. We describe the topology by Dierolf. Let $\mathcal{N}$ be the family of all subsets $N$ of $E^{\prime}$ such that $N$ is $\sigma\left(E^{\prime}, E\right)$ bounded and for every linear continuous mapping

$$
T:\left(E^{\prime}, \sigma\left(E^{\prime}, E\right)\right) \rightarrow\left(l^{1}, \sigma\left(l^{1}, l^{\infty}\right)\right) .
$$

$T(N)$ is relatively compact in $\left(l^{1},\|\cdot\|_{1}\right)[3]$. The Dierolf topology $\delta_{2}\left(E, E^{\prime}\right)$ on $E$ is defined to be the polar topology on $E$ of uniform convergence on the elements of $\mathcal{N}$ (see [3: Corollary 2.4] for properties of the topology).

The analogues of Propositions 2.6 and 2.7 and Corollary 2.8.hold for $\delta_{2}$.

Proposition 2.10. $\delta_{2}\left(E, E^{\prime}\right)$ is a Hellinger-Töplitz topology.

Proposition 2.11. For $t \in l^{\infty}$,

$$
\delta_{2}\left(l^{\infty}, l^{1}\right)-\lim _{n} \sum_{k=1}^{n} t_{k} e^{k}=t
$$

uniformly for $\|t\|_{\infty} \leq 1$. In particular, $\left(l^{\infty}, \delta_{2}\left(l^{\infty}, l^{1}\right)\right)$ is an AK-space.

Corollary 2.12 (Orlicz-Pettis). If $\sum x_{k}$ is $\sigma\left(X, X^{\prime}\right) l^{\infty}$ multiplier convergent, then

$$
\delta_{2}\left(X, X^{\prime}\right)-\lim _{n} \sum_{k=n}^{\infty} t_{k} x_{k} \doteq \sum_{k=1}^{\infty} t_{k} x_{k}
$$

uniformly for $t \in l^{\infty}$ with $\|t\|_{\infty} \leq 1$. In particular, $\sum x_{k}$ is $\delta_{2}\left(X, X^{\prime}\right) l^{\infty}$ multiplier convergent.

The uniform convergence conclusion in the case of bounded multiplier convergent series has been previously observed (see, for example, [10: Corollary 8.2.7]).

In contrast to Corollary 2.4 the conclusions to Corollaries 2.8 and 2.11 cannot be improved to convergence in the strong topology. 
Example 2.13. The series $\sum e^{k}$ in $l^{\infty}\left(m_{0}\right)$ is $\sigma\left(l^{\infty}, l^{1}\right)\left(\sigma\left(m_{0}, l^{1}\right)\right) l^{\infty}\left(m_{0}\right)$ multiplier convergent but not $\beta\left(l^{\infty}, l^{1}\right)=\|\cdot\|_{\infty}\left(\beta\left(m_{0}, l^{1}\right)=\|\cdot\|_{\infty}\right)$ convergent.

Although we cannot, in general, show that weakly multiplier convergent series are strongly multiplier convergent series, the "partial sums" of such series are often strongly bounded.

Recall that a sequence space $\lambda$ is an $A B$-space if the coordinate functions $t \rightarrow t_{k}$ are continuous and if $\left\{\sum_{k=1}^{n} t_{k} e^{k}: n \in \mathbb{N}\right\}$ is bounded in $\lambda$ for each $t \in \lambda$ [12: Definition 10.2.1].

Proposition 2.14. Let $\lambda$ be a barrelled $A B$-space with $\lambda^{\beta} \subset \lambda^{\prime}$. If $\sum x_{k}$ is $\sigma\left(X, X^{\prime}\right)$ $\lambda$ multiplier convergent and $B \subset \lambda$ is bounded, then the set of "partial sums"

$$
\mathcal{S}(B)=\left\{\sum_{k=1}^{n} t_{k} x_{k}: n \in \mathbb{N} \text { and } t \in B\right\}
$$

is $\beta\left(X, X^{\prime}\right)$ bounded.

Proof. Let $P_{n}: \lambda \rightarrow \lambda$ be the section map $P_{n} t=\sum_{k=1}^{n} t_{k} e^{k}$. Then $\left\{P_{n}: n \in \mathbb{N}\right\}$ is pointwise bounded on $\lambda$ by the $A B$-assumption and is, hence, equicontinuous by the barrelledness assumption. Since $\lambda$ has the $\beta\left(\lambda, \lambda^{\prime}\right)$ topology, $\left\{P_{n} B: n \in \mathbb{N}\right\}$ is $\beta\left(\lambda, \lambda^{\prime}\right)$ bounded and, hence, $\beta\left(\lambda, \lambda^{\beta}\right)$ bounded since $\lambda^{\beta} \subset \lambda^{\prime}$. By Corollary $2.2 \mathcal{S}(B)=\left\{S P_{n} B\right.$ : $n \in \mathbb{N}\}$ is $\beta\left(X, X^{\prime}\right)$ bounded

Example 2.15. Since $l^{\infty}$ and $m_{0}$ are a barrelled $A B$-space under the sup-norm and $\left(l^{\infty}\right)^{\beta}=l^{1} \subset\left(l^{\infty}\right)^{\prime}=b a$ and $m_{0}^{\beta}=l^{1} \subset\left(m_{0}\right)^{\prime}=b a[4:$ p. 69], Propositon 2.14 is applicable to both weak subseries and bounded multiplier convergent series. Likewise, Proposition 2.14 is applicable to all of the spaces listed in Example 2.5. Proposition 2.14 is also applicable to the spaces $b s, b v$, and $c$ whereas Corollary 2.4 is not (see [4: pp. $68-69$ ] for the topological and $\beta$-duals of these spaces).

\section{References}

[1] Bennett, G.: Some inclusion theorems for sequence spaces. Pac. J. Math. 64 (1973), 17 $-30$.

[2] Bennett, G. and N. Kalton: Inclusion theorems for K-spaces. Can. J. Math. 25 (1973), $511-524$.

[3] Dierolf, P.: Theorems of the Orlicz-Pettis type for locally convex spaces. Man. Math. 20 (1977), 73 - 94.

[4] Kamthan, P. and M. Gupta: Sequence Spaces and Series. New York: Marcel Dekker 1981.

[5] Köthe, G.: Topological Vector Spaces. Vol. 1. New York: Springer Verlag 1969.

[6] Li Ronglu, Cui Changri and Min Hyung Cho: An invariant with respect to all admissible $\left(X, X^{\prime}\right)$-polar topologies. Chinese Ann. Math. 3 (1998) (to appear).

[7] McArthur, C.: On a theorem of Orlicz and Pettis. Pac. J. Math. 22 (1967), $297-302$.

[8] Ruckle, W. and S. Saxon: Generalized sectional convergence. J. Math. Anal. Appl. 193 (1995), $680-705$. 
[9] Swartz, C.: An Introduction to Functional Analysis. New York: Marvel Dekker 1992.

[10] Swartz, C.: Infinite Matrices and the Gliding Hump. Singapore: World Scientific 1996.

[11] Wilansky, A.: Modern Methods in Topological Vector Spaces. New York: McGraw-Hill 1978.

[12] Wilansky, A.: Summability through Functional Analysis. Amsterdam: North-Holland 1984.

[13] Wu Junde and Li Ronglu: Basic properties of locally convex A-spaces. Studia Sci. Math. Hungar. (to appear).

Received 05.05.1998 